\title{
Risk factors for lymph node metastasis and the impact of adjuvant chemotherapy on ductal carcinoma in situ with microinvasion: a population-based study
}

This article was published in the following Dove Press journal: OncoTargets and Therapy

\section{Cong Chen ${ }^{1,2}$ \\ Shumin Huang ${ }^{3}$ \\ Aihua Huang ${ }^{2,4}$ \\ Yunlu Jia ${ }^{1,2}$ \\ Ji Wang ${ }^{1,2}$ \\ Zeqin Zhang ${ }^{1,2}$ \\ Misha Mao',2 \\ Linbo Wang ${ }^{1,2}$ \\ Jichun Zhou' ${ }^{1,2}$}

'Department of Surgical Oncology, Sir Run Run Shaw Hospital, Zhejiang University School of Medicine, Hangzhou, China; ${ }^{2}$ Biomedical Research Center and Key Laboratory of Biotherapy of Zhejiang Province, Hangzhou, China; ${ }^{3}$ The Children's Hospital, Zhejiang University School of Medicine, Hangzhou, China; ${ }^{4}$ Department of Pathology, Sir Run Run Shaw Hospital, Zhejiang University School of Medicine, Hangzhou, China
Correspondence: Linbo Wang; Jichun Zhou

Department of Surgical Oncology, Sir Run Run Shaw Hospital, Zhejiang University School of Medicine, No 3 Eastern Qingchun Road, Hangzhou, Zhejiang 310016, China Email linbowang@zju.edu.cn; jichun-zhou@zju.edu.cn
Background: Ductal carcinoma in situ with microinvasion (DCISM) represents $\sim 1 \%$ of all breast cancer cases. Risk factors for lymph node (LN) metastasis and appropriate adjuvant therapy for DCISM are still widely debated.

Methods: We retrieved DCISM data from the National Cancer Institute's Surveillance, Epidemiology, and End Results registry database (1998-2013). Chi-squared tests and logistic regression models were applied to investigate the potential risks of LN metastasis. Univariate and multivariate Cox proportional hazards regressions were performed to estimate the prognostic factors of DCISM. Survival outcomes were estimated using the Kaplan-Meier method. A 1:1 propensity score matching was used to minimize potential bias.

Results: Overall, 6,219 patients with DCISM met our inclusion criteria. Younger age and higher grade disease were identified as risk factors for LN metastasis. In the multivariable analysis, LN metastasis and chemotherapy were prognostic factors for worse overall survival and breast cancer-specific survival. Furthermore, propensity score matching and subgroup analysis showed that chemotherapy may not be effective for DCISM patients.

Conclusion: Younger patients with high-grade disease tend to have LN involved in DCISM. Adjuvant chemotherapy might not be necessary for patients with DCISM.

Keywords: SEER database, breast cancer, ductal carcinoma in situ with microinvasion, adjuvant chemotherapy, lymphatic metastasis

\section{Introduction}

Ductal carcinoma in situ with microinvasion (DCISM) is a rare pathologic entity accounting for $\sim 0.6 \%-3.4 \%$ of all breast cancer cases. ${ }^{1,2}$ The specific definitions of this entity have varied in the past decades, and the seventh edition of the American Joint Committee on Cancer Cancer Staging Manual defines microinvasion as not exceeding $1 \mathrm{~mm}$ in size. ${ }^{3}$ With the widespread use of mammography in screening, increasing numbers of DCISM are now being detected and diagnosed.

The presence of lymph node (LN) metastasis has been reported to range between $0 \%$ and $25 \% .^{4-9}$ Although many pathological factors might be associated with LN metastasis, including lymph angiogenesis, ${ }^{7}$ high-grade disease, ${ }^{10}$ larger ductal carcinoma in situ (DCIS) lesion, ${ }^{11}$ and younger age,${ }^{9}$ the reliability of these conclusions should be interpreted carefully due to the small number of studies.

DCISM presents a therapeutic conundrum. The prognosis falls somewhere between invasive cancer and DCIS, but the specific prognosis of individuals is 
unclear, and risk stratification based on retrospective reports has been difficult due to the overall rarity of this entity. ${ }^{12}$ Although classified as invasive cancer, DCISM has an excellent prognosis compared with DCIS. ${ }^{4}$ Unfortunately, an agreement has not been reached on the standard therapy for DCISM. Adjuvant chemotherapy is generally recommended for invasive ductal carcinoma (IDC) with LN metastasis, while it remains undetermined whether DCISM with LN involvement should receive chemotherapy.

Based on retrieved DCISM data from the National Cancer Institute's Surveillance, Epidemiology, and End Results (SEER) registry database (1998-2013), we designed a population-based study to identify risk factors for LN metastasis in DCISM and, more importantly, weigh the impact of adjuvant chemotherapy on DCISM.

\section{Methods}

\section{Patient selection}

Data were extracted from the National Cancer Institute's SEER program between 1998 and 2013. The SEER project is a United States population-based cancer registry that began in 1973 and now includes 18 registries across the United States, covering $\sim 28 \%$ of the American population.

We focused on cases diagnosed between 1998 and 2013 with microscopically confirmed stage T1mic, and we selected patients aged between 20 and 70 years old. Information about patients with infiltrating duct carcinoma (code 8500/3) was referenced to the International Classification of Diseases for Oncology, version 3. Patients who were identified at autopsy, with death certificate only, no surgery treatment, or incomplete survival times were excluded from our study. Tumor demographics included grade (grade I: well differentiated/grade II: moderately differentiated/grade III: poorly differentiated/grade IV: undifferentiated/unknown), hormone status (estrogen receptor [ER] positive/negative/unknown, progesterone receptor [PR] positive/negative/unknown), and human epidermal growth factor receptor 2 (HER2) status (positive/negative/ unknown). Treatment characteristics included surgery (lumpectomy/mastectomy), chemotherapy, and radiotherapy. Additionally, as SEER did not report the type of LN surgery, we used the number of LNs excised as an alternative. We define patients with 1-5 LNs removed as sentinel lymph node biopsy (SLNB) and $>5$ LNs removed as axillary lymph node dissection (ALND), as previous studies reported. ${ }^{13,14}$ A total of 6,219 female patients with DCISM met our inclusion criteria.

\section{Statistical analysis}

The characteristics of the two groups of patients (LN positive vs LN negative) were compared using Pearson's chi-squared test or Fisher's exact test. Logistic regression analysis was further used to identify the independent factors associated with LN metastasis. Overall survival (OS) was calculated as the time from the date of diagnosis to the date of death from any cause. Breast cancer-specific survival (BCSS) was measured from the date of diagnosis to the date of death owing to breast cancer. Survival rates were assessed using the Kaplan-Meier method and compared using the log-rank test. Furthermore, we used the Cox proportional hazards model to calculate HR and $95 \%$ CI for the prognostic factors of DCISM. To minimize group differences, a 1:1 propensity score matching was used.

All statistical analyses were performed using SPSS statistical software, version 22.0 (IBM Corporation, Armonk, NY, USA), and a $P$-value $<0.05$ was considered statistically significant.

\section{Ethics statement}

This was a retrospective analysis using data from the SEER database, which is a public health database. The data released by the SEER database were publicly accessible to applicants and did not require informed patient consent for use.

\section{Results}

\section{Patient and treatment characteristics}

The characteristics of all patients are listed in Table S1. The mean (range) age at diagnosis of DCISM was 53.8 (20-70) years. Overall, the majority $(74.5 \%)$ of patients were white. More than half $(58.4 \%)$ of the patients had low-grade disease (grade I+II). Moreover, $68.7 \%$ of patients had positive ER expression, and 55.2\% had positive PR expression. Only $7.8 \%$ of patients had LN metastasis. As SEER began recording HER2 status in 2010, there was little information available for this subgroup.

For surgical treatment, SLNB was performed in 4,102 patients $(66.4 \%)$, lumpectomy was performed in 3,388 patients $(54.5 \%)$, corresponding to 1,496 patients $(24.2 \%)$ with ALND and 2,831 patients (45.5\%) with mastectomy. In addition, $46.9 \%$ of patients underwent radiotherapy, and a small number of patients $(9.8 \%)$ received chemotherapy.

\section{$\mathrm{LN}$ positive vs $\mathrm{LN}$ negative}

We then divided DCISM into two groups: LN positive and LN negative. There was no difference in ER $(P=0.396)$, PR $(P=0.414)$, and HER2 $(P=0.126)$ expression, but much 
more high-grade diseases and young patients were observed in the LN-positive group (50.9\% vs $41.5 \%, P<0.001$; $30.1 \%$ vs $17.4 \%, P<0.001$, respectively). Age at diagnosis, ethnicity, grade, hormone receptor status, and HER2 status were analyzed in the binary logistic regression model. Using the multivariate analysis, younger age and higher grade disease were found to be independent risk factors responsible for $\mathrm{LN}$ metastasis. The results of the univariate and multivariate analyses are summarized in Tables 1 and 2 .

\section{Survival outcomes}

The median follow-up was 76 months (range, 1-170 months). There were 271 deaths in all and 90 breast cancer-specific deaths. The 10-year OS and BCSS of all patients were $93.8 \%$ and $97.7 \%$, respectively. The 10-year OS for LN-negative patients was $94.7 \%$, compared to $87.4 \%$ in the $\mathrm{LN}$-positive group (Figure 1A; $P<0.001$ ), and the 10 -year BCSS for LN-negative patients was $98.4 \%$, compared to $91.3 \%$ in the LN-positive group (Figure 1B; $P<0.001$ ).

Table I Patient characteristics stratified by lymph node status and univariate analysis of risk factors of lymph nodes metastasis

\begin{tabular}{|c|c|c|c|c|c|}
\hline Variables & LN positive & $\%$ & LN negative & $\%$ & $P$-value \\
\hline Age (years) & & & & & $<0.001$ \\
\hline$<45$ & $|3|$ & 30.1 & 905 & 17.4 & \\
\hline$\geq 45$ & 304 & 69.9 & 4,288 & 82.6 & \\
\hline Ethnicity & & & & & $<0.001$ \\
\hline White & 320 & 73.6 & 3,863 & 74.4 & \\
\hline Black & 77 & 17.7 & 592 & II.4 & \\
\hline Other & 38 & 8.7 & 738 & 14.2 & \\
\hline Grade & & & & & 0.001 \\
\hline I+II & 158 & 49.1 & 2,045 & 58.5 & \\
\hline III+IV & 164 & 50.9 & $\mathrm{I}, 448$ & 41.5 & \\
\hline Unknown & 113 & & 1,700 & & \\
\hline ER status & & & & & 0.396 \\
\hline Negative & 132 & 33.8 & I,4II & 31.7 & \\
\hline Positive & 259 & 66.2 & 3,044 & 68.3 & \\
\hline Unknown & 44 & & 738 & & \\
\hline PR status & & & & & 0.414 \\
\hline Negative & $|8|$ & 47.3 & $\mathrm{I}, 948$ & 45.1 & \\
\hline Positive & 202 & 52.7 & 2,372 & 54.9 & \\
\hline Unknown & 52 & & 873 & & \\
\hline HER2 status & & & & & 0.126 \\
\hline Negative & 54 & 52.9 & 693 & 60.7 & \\
\hline Positive & 48 & 47.1 & 449 & 39.3 & \\
\hline Unknown & 333 & & 4,051 & & \\
\hline Radiotherapy & & & & & $<0.001$ \\
\hline No & 282 & 65.1 & 2,750 & 53.2 & \\
\hline Yes & $|5|$ & 34.9 & 2,420 & 46.8 & \\
\hline Unknown & 2 & & 23 & & \\
\hline Chemotherapy & & & & & $<0.001$ \\
\hline No & 130 & 29.9 & 4,921 & 94.8 & \\
\hline Yes & 305 & 70.1 & 272 & 5.2 & \\
\hline LN surgery & & & & & $<0.001$ \\
\hline SLNB & $|2|$ & 27.9 & 3,981 & 77.1 & \\
\hline ALND & 313 & 72.1 & $\mathrm{I}, \mathrm{I} 82$ & 22.9 & \\
\hline Unknown & 1 & & 30 & & \\
\hline Breast surgery & & & & & $<0.001$ \\
\hline Lumpectomy & 112 & 25.7 & 2,813 & 54.2 & \\
\hline Mastectomy & 323 & 74.3 & 2,380 & 45.8 & \\
\hline Vital status & & & & & $<0.001$ \\
\hline Alive & 386 & 88.7 & 5,010 & 96.5 & \\
\hline Dead of other cause & 19 & 4.4 & 135 & 2.6 & \\
\hline Breast cancer-specific death & 30 & 6.9 & 48 & 0.9 & \\
\hline
\end{tabular}

Abbreviations: ALND, axillary lymph node dissection; ER, estrogen receptor; HER2, human epidermal growth factor receptor 2; LN, lymph node; PR, progesterone receptor; SLNB, sentinel lymph node biopsy. 
Table 2 Multivariable regression analysis evaluating factors associated with lymph nodes metastasis

\begin{tabular}{l|l|l}
\hline Variables & HR $(95 \% \mathbf{C l})$ & $P$-value \\
\hline Age (years) & & $<0.00 \mathrm{I}$ \\
$<45$ & Reference & \\
$\geq 45$ & $0.496(0.398-0.617)$ & \\
Ethnicity & & $0.00 \mathrm{I}$ \\
White & Reference & \\
Black & $1.547(\mathrm{I} .186-2.018)$ & \\
Grade & & 0.008 \\
I+II & Reference & \\
III+IV & $1.385(1.088-1.763)$ & \\
\hline
\end{tabular}

\section{Prognostic factor}

In the univariate analysis, age at diagnosis, ethnicity, tumor grade, PR status, LN status, radiotherapy, chemotherapy, and surgery treatment were significantly associated with OS or (and) BCSS.

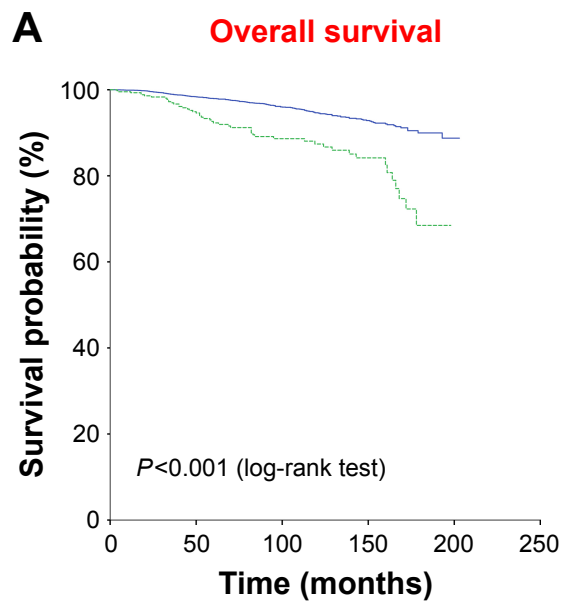

B Breast cancer-
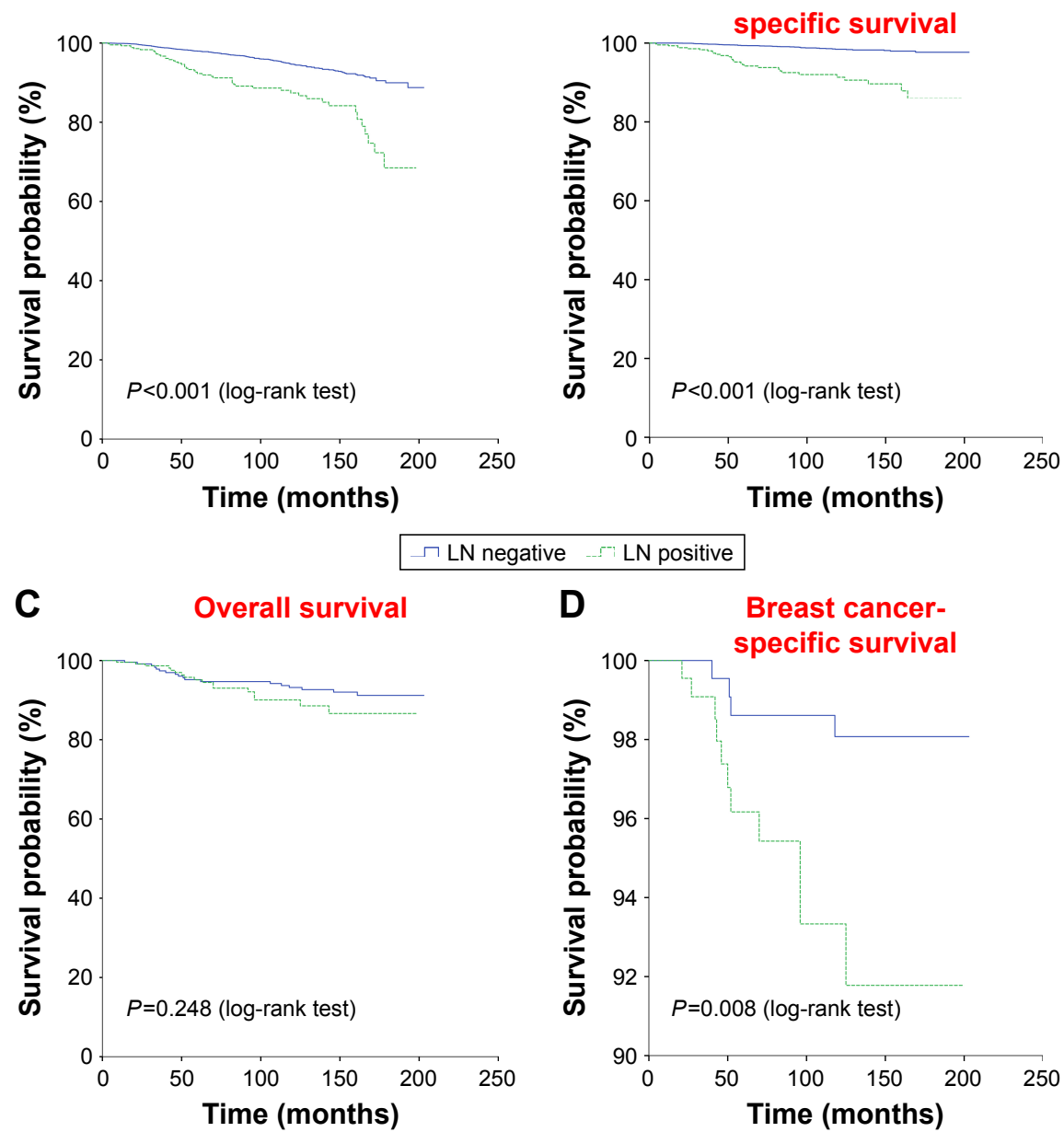

\section{LN positive}

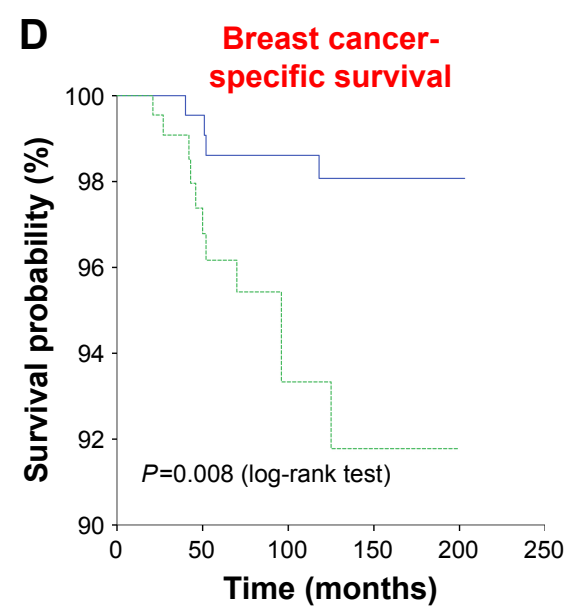

In the multivariate analysis, older age (HR 1.762, 95\% CI 1.228-2.529, $P=0.002$ ), black women (HR 2.188, 95\% CI 1.645-2.910, $P<0.001)$, LN metastasis (N2: HR 3.697, 95\% CI 1.818-7.519, $P<0.001$; N3: HR 6.846, 95\% CI 1.989-23.568, $P=0.002$ ), and chemotherapy (HR 1.701, 95\% CI 1.133-2.555, $P=0.010)$ were associated with worse OS. However, positive PR expression (HR 0.559, 95\% CI $0.385-0.811, P=0.002$ ) and radiotherapy (HR 0.597, 95\% CI $0.426-0.837, P=0.003$ ) were significantly good predictors of breast cancer OS. Moreover, black women (HR 2.081, 95\% CI 1.243-3.484, $P=0.005)$, LN metastasis (N2: HR 4.732, 95\% CI 1.977-11.324, $P<0.001$; N3: HR 7.139, 95\% CI 1.449-35.161, $P=0.016$ ), chemotherapy (HR 3.938, 95\% CI 2.168-7.153, $P<0.001$ ), and mastectomy (HR 2.163, 95\% CI 1.182-3.956, $P=0.012$ ) were related with worse BCSS. As the majority of HER2 variables was not known (SEER database collected HER2 information after 2010), the results

$\checkmark$ No chemotherapy $\quad$ Chemotherapy

Figure I DCISM survival outcomes estimated by the Kaplan-Meier method.

Notes: Breast cancer overall survival curves $(\mathbf{A})$ and breast cancer-specific survival curves (B) for patients with DCISM, stratified by LN status. Breast cancer overall survival (C) and breast cancer-specific survival (D) stratified by chemotherapy after propensity score matching.

Abbreviations: DCISM, ductal carcinoma in situ with microinvasion; LN, lymph node. 
of HRs were insignificant in the univariate and multivariate analyses. The results of univariate and multivariate analyses are listed in Table 3.

\section{Propensity score matching}

Patients who received chemotherapy may have been subject to some selection bias. To further adjust for potential baseline bias and confounders, propensity score matching was carried out. Moreover, to minimize confounders, we also excluded some unavailable or unknown information, such as unknown ER status, PR status, LN stage, and radiotherapy. Finally, a total of 3,198 patients with complete information were available. All matching variables were balanced between the two groups (chemotherapy vs no chemotherapy); details of the original, unmatched cohort, and propensity-matched cohort are shown in Table 4. After matching, survival analysis and log-rank testing revealed worse BCSS in the chemotherapy-treated group than in the no chemotherapy group (Figure 1D; $P=0.008$ ), but there was no statistical significance for OS (Figure 1C; $P=0.248$ ).

In the LN-negative subgroup, the same methods were implemented as earlier. Finally, 2,916 LN-negative patients with complete information were available; details of the original, unmatched cohort, and propensity-matched cohort are shown in Table S2. After matching, survival analysis showed no statistical significance in the two groups (chemotherapy vs no chemotherapy) for OS (Figure $\mathrm{S} 1 \mathrm{~A} ; P=0.324$ ) and BCSS (Figure S1B; $P=0.121$ ). Unfortunately, due to the limited number, we could not apply propensity score matching to the LN-positive subgroup.

\section{Subgroup analysis}

Univariate and multivariate Cox proportional hazards regression models were also employed in the LN-negative and LN-positive subgroups. For the LN-negative subgroup, chemotherapy was an independent factor for worse OS (HR 2.119, 95\% CI 1.311-3.424, $P=0.002$ ) and BCSS (HR 4.631, 95\% CI 2.253-9.114, $P<0.001$ ), as shown in Table S3. For the LN-positive subgroup, chemotherapy was not associated with better OS $(P=0.559)$ and BCSS $(P=0.288)$, as shown in Table S4.

\section{Discussion}

DCISM is a disease entity that is not fully characterized, in contrast to DCIS and IDC. The clinical-pathological characteristics are unclear, and the optimal treatment is controversial due to limited information. Although there have already been two studies about DCISM based on the SEER database, ${ }^{15,16}$ they focused on comparing DCISM with

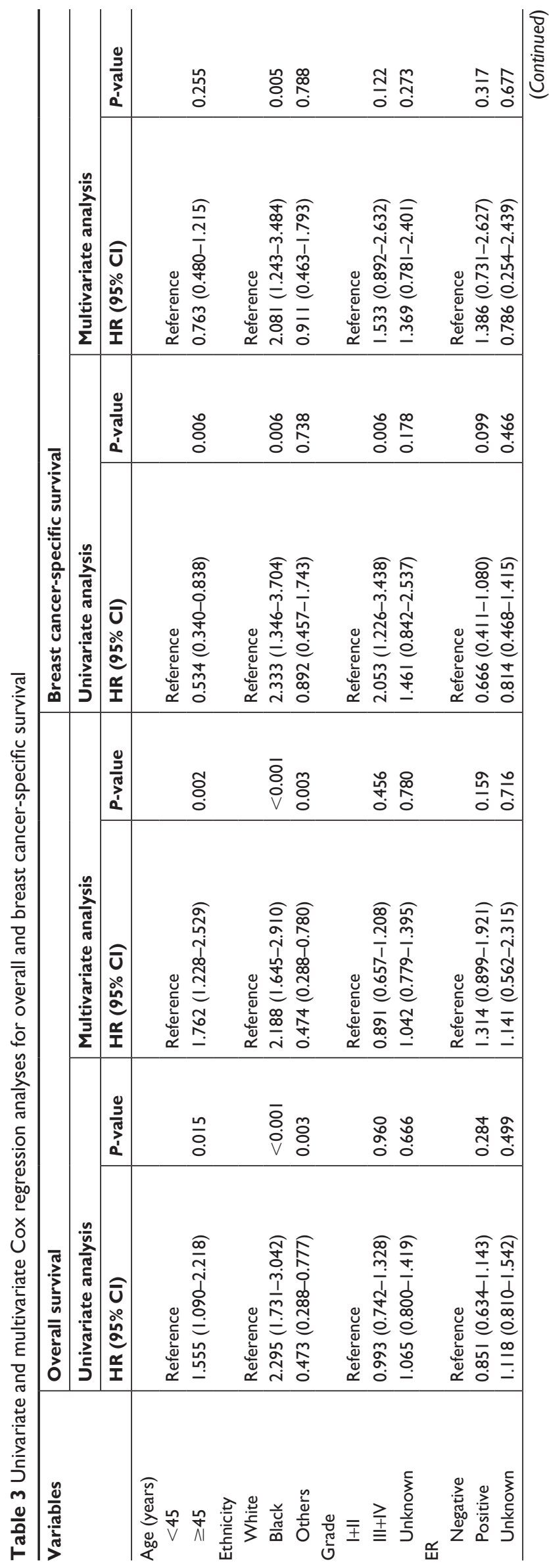




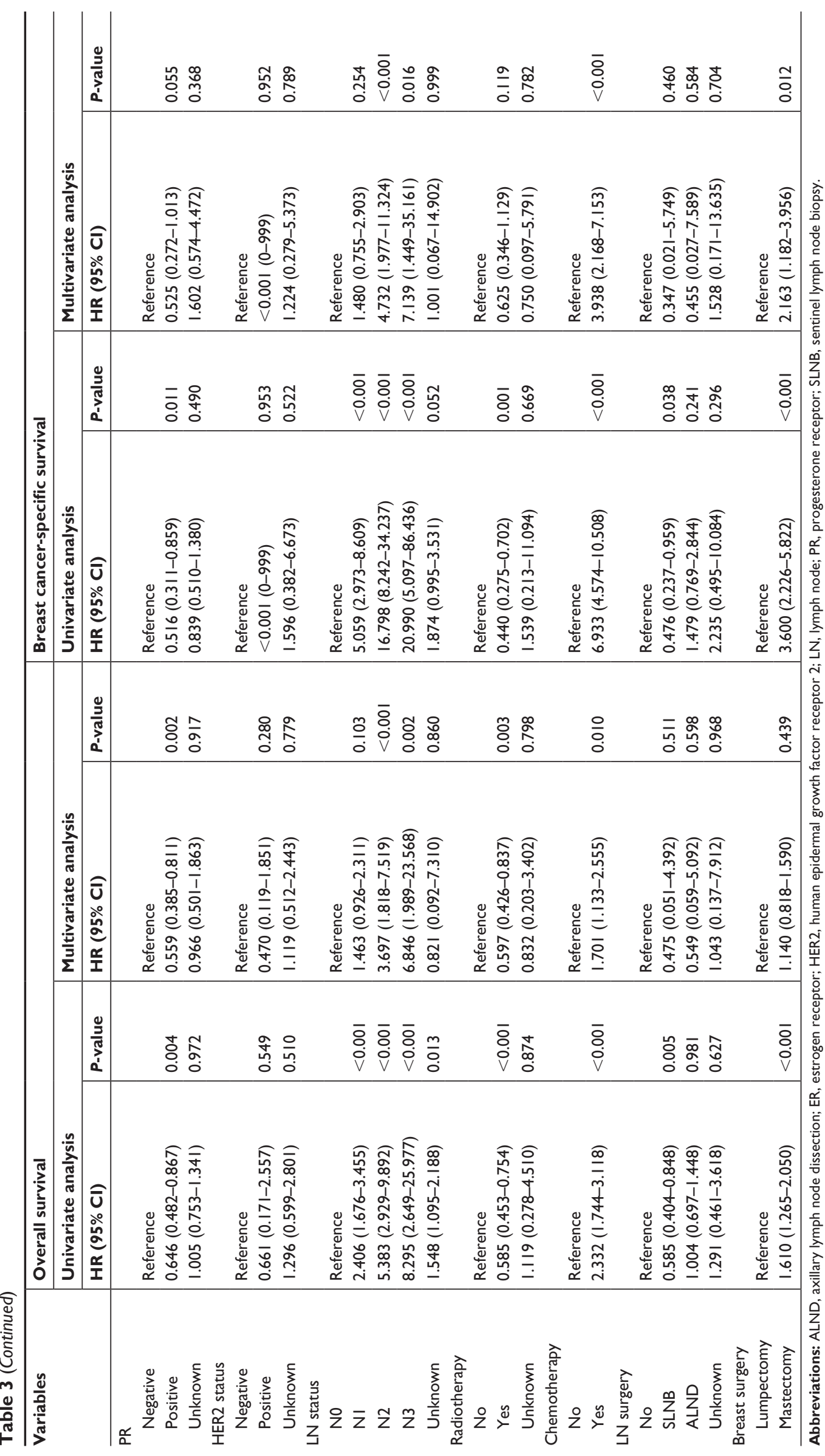


Table 4 Baseline characteristics of DCISM patients treated with or without chemotherapy

\begin{tabular}{|c|c|c|c|c|c|c|c|c|c|c|}
\hline \multirow[t]{3}{*}{ Variables } & \multicolumn{4}{|c|}{ Original, unmatched cohort } & \multirow[t]{3}{*}{$P$-value } & \multicolumn{4}{|c|}{ Propensity-matched cohort } & \multirow[t]{3}{*}{$P$-value } \\
\hline & \multicolumn{2}{|c|}{ No chemotherapy } & \multicolumn{2}{|c|}{ Chemotherapy } & & \multicolumn{2}{|c|}{ No chemotherapy } & \multicolumn{2}{|c|}{ Chemotherapy } & \\
\hline & No & $\%$ & No & $\%$ & & No & $\%$ & No & $\%$ & \\
\hline Age (years) & & & & & $<0.001$ & & & & & 0.450 \\
\hline$<45$ & 470 & 16.8 & 120 & 30.3 & & 53 & 22.6 & 60 & 25.5 & \\
\hline$\geq 45$ & 2,332 & 83.2 & 276 & 69.7 & & 182 & 77.4 & 175 & 74.5 & \\
\hline Ethnicity & & & & & 0.469 & & & & & 0.849 \\
\hline White & 2,092 & 74.7 & 291 & 73.5 & & 174 & 74.0 & 177 & 75.3 & \\
\hline Black & 331 & 11.8 & 55 & 13.9 & & 30 & 12.8 & 26 & II.I & \\
\hline Other & 379 & 13.5 & 50 & 12.6 & & 31 & 13.2 & 32 & 13.6 & \\
\hline Grade & & & & & $<0.001$ & & & & & 0.517 \\
\hline I+II & 1,692 & 60.4 & 169 & 42.7 & & 110 & 46.8 & 103 & 43.8 & \\
\hline III+IV & 1,110 & 39.6 & 227 & 57.3 & & 125 & 53.2 & 132 & 56.2 & \\
\hline ER status & & & & & $<0.001$ & & & & & 0.782 \\
\hline Negative & 799 & 28.5 & 184 & 46.5 & & 110 & 46.8 & 113 & 48.1 & \\
\hline Positive & 2,003 & 71.5 & 212 & 53.5 & & 125 & 53.2 & 122 & 51.9 & \\
\hline PR status & & & & & $<0.001$ & & & & & 0.396 \\
\hline Negative & $\mathrm{I}, \mathrm{I} 88$ & 42.4 & 231 & 58.3 & & 137 & 58.3 & 146 & 62.1 & \\
\hline Positive & $|, 6| 4$ & 57.6 & 165 & 41.7 & & 98 & 41.7 & 89 & 37.9 & \\
\hline LN status & & & & & $<0.001$ & & & & & 0.797 \\
\hline No & 2,728 & 97.4 & 188 & 47.5 & & 183 & 77.9 & 183 & 77.9 & \\
\hline $\mathrm{NI}$ & 70 & 2.5 & 165 & 41.7 & & 48 & 20.4 & 45 & 19.1 & \\
\hline N2 & 4 & 0.1 & 35 & 8.8 & & 4 & 1.7 & 6 & 2.6 & \\
\hline N3 & 0 & 0 & 8 & 2.0 & & 0 & 0 & 1 & 0.4 & \\
\hline Radiotherapy & & & & & 0.035 & & & & & 0.778 \\
\hline No & $\mathrm{I}, 448$ & 51.7 & 227 & 57.3 & & 137 & 58.3 & 140 & 59.6 & \\
\hline Yes & $\mathrm{I}, 354$ & 48.3 & 169 & 42.7 & & 98 & 41.7 & 95 & 40.4 & \\
\hline LN surgery & & & & & $<0.001$ & & & & & 0.115 \\
\hline SLNB & 2,201 & 78.6 & 161 & 40.7 & & 122 & 51.9 & 139 & 59.1 & \\
\hline ALND & 601 & 21.4 & 235 & 59.3 & & 113 & 48.1 & 96 & 40.9 & \\
\hline Breast surgery & & & & & $<0.001$ & & & & & 0.578 \\
\hline Lumpectomy & $\mathrm{I}, 555$ & 55.5 & 139 & 35.1 & & 108 & 46.0 & 102 & 43.4 & \\
\hline Mastectomy & $\mathrm{I}, 247$ & 44.5 & 257 & 64.9 & & 127 & 54.0 & 133 & 56.6 & \\
\hline
\end{tabular}

Abbreviations: ALND, axillary lymph node dissection; DCISM, ductal carcinoma in situ with microinvasion; ER, estrogen receptor; LN, lymph node; PR, progesterone receptor; SLNB, sentinel lymph node biopsy.

DCIS and IDC and did not analyze the therapy strategies, such as chemotherapy and surgical options. We first divided the DCISM into two groups (LN positive vs LN negative) to explore the difference between the two groups and identify the potential risks for LN metastasis. To our knowledge, this population-based study is the first to identify predictive markers for LN metastasis and report the potential influence of adjuvant chemotherapy on DCISM.

There is a potential risk of LN metastasis in DCISM. Some studies reported that it was valuable to perform SLNB routinely, ${ }^{9}$ while others had different perspectives and stated that SLNB should be individualized. ${ }^{17}$ These debates raised the question of whether there were subgroups of patients who were at high risk of $\mathrm{LN}$ metastasis.

In our study, $7.8 \%$ of patients had LN metastasis. Importantly, our study identified that younger age and higher grade disease were risk factors for LN involvement. In a study of 81 patients with DCISM, Gray et al suggested that extensive size of DCIS ( $>5 \mathrm{~cm})$ and multifocal microinvasion were associated with LN metastasis. ${ }^{5}$ Wasserberg et al revealed that nuclear grade, comedonecrosis, and DCIS-involved ducts might predict LN metastasis. However, only high-grade status was a significant factor for LN metastasis in T1a breast cancer. ${ }^{10}$ Kapoor et al analyzed 45 patients with DCISM and found that the LN was involved in nine patients. In the multivariate analysis, only negative ER status $(P<0.02)$ was a risk factor for LN metastasis rather than high-grade disease, comedonecrosis, lymphovascular invasion, DCIS size, multifocal microinvasion, and HER 2 status. ${ }^{18}$ However, several studies have proposed contrary conclusions. Guth et al proposed that comedonecrosis, nuclear grade, multifocal DCIS, and hormone receptor status might not predict LN involvement, but patients with $\mathrm{LN}$ positivity tended to be younger, ${ }^{9}$ which is consistent with our current study. Pimiento et al studied the correlation between clinical-pathological features and axillary LN metastasis in 90 female patients 
with DCISM. Unfortunately, this study failed to identify any significant predictive factor. ${ }^{19}$ In addition, by studying records from a prospective institutional database, Matsen et al investigated 414 patients with DCISM and suggested that multifocal microinvasion may not be correlated with LN involvement. ${ }^{12}$ All these published studies were limited by small sample size, and the reliability of their conclusions is therefore not confirmed. Larger studies are urgently needed to identify the relevant predictive factors of $\mathrm{LN}$ metastasis in patients with DCISM.

To take it further, the significance of $\mathrm{LN}$ involvement in DCISM is another topic of interest - specifically, the predictive value of $\mathrm{LN}$ involvement on locoregional recurrence and distant metastasis. Unfortunately, the SEER database cannot provide this critical information.

The other interesting finding from our current study is that adjuvant chemotherapy might not be necessary for DCISM. Especially for the subgroup of LN-negative patients, chemotherapy is even a risk prognostic factor for worse OS (HR 2.119, 95\% CI 1.311-3.424, $P=0.002$ ) and BCSS (HR 4.631, 95\% CI 2.253-9.114, $P<0.001$ ). Although, we could not apply propensity score matching to investigate the effect of chemotherapy in the LN-positive subgroup (it may be underpowered to do so), univariate and multivariate Cox proportional hazards regression models suggested that chemotherapy was not associated with better OS $(P=0.559)$ and BCSS $(P=0.288)$.

Because chemotherapy is seldom needed for DCISM, the impact of chemotherapy on this uncommon entity is unclear until now. Fang et al presented 84 DCISM patients, 16 of whom were treated with chemotherapy and trastuzumab. After univariate and multivariate analyses, HER2 status was an independent predictor for worse disease-free survival with a median follow-up of 31 months, and they suggested that chemotherapy and target therapy in patients with HER2-positive disease seemed to be reasonable. ${ }^{20}$ Matsen et al reported that patients with LN involvement and multifocal microinvasion were more likely to receive chemotherapy. ${ }^{12}$ Lyons et al found 14 patients with positive sentinel LN (SLN) among 112 patients with DCISM, and adjuvant chemotherapy was given to all patients with macrometastasis. With a median follow-up of 6 years, there were five local recurrences. It is worth discussing that all five recurrences were observed in patients with negative SLNs. Thus, the authors concluded that DCISM patients with SLN macrometastasis would benefit from adjuvant chemotherapy. ${ }^{21}$ On the other hand, regardless of LN metastasis, DCISM patients also reported excellent prognosis. ${ }^{22,23}$ Therefore, adjuvant therapy may be unnecessary for this already excellent prognosis disease. ${ }^{24}$ Niu et al found no statistical significance in the 5-year disease-free survival or OS between chemotherapy and nonchemotherapy groups but it might improve the outcomes of ER-negative/PR-negative DCISM patients. ${ }^{25}$ According to the latest guidelines, including National Comprehensive Cancer Network guidelines, chemotherapy was routinely recommended for breast cancer patients with $\mathrm{LN}$ involvement. ${ }^{26}$ However, it is unclear whether patients were potentially overtreated or the relatively rare recurrence in the node-positive group was due to adjuvant chemotherapy. ${ }^{27}$ The MINDACT trial is a phase III trial comparing the 70-gene signature with the commonly used clinicopathologic criteria in selecting patients for adjuvant chemotherapy in breast cancer with $0-3$ positive nodes. It is worth noting that early results from the MINDACT trial suggest that the 70-gene signature can help avoid chemotherapy in certain patients regardless of larger tumor size and nodal status, without compromising the outcome. ${ }^{28}$ Based on these data, in the era of precision medicine, clinicians should consider the biology of tumors while making clinical decisions. The costs and benefits of chemotherapy should be exactly weighted in DCISM patients. In addition, patients who can derive the most benefit from chemotherapy should be further identified.

Our present study has several limitations. First, when both in situ and invasive components are present in a tumor, the SEER database records only the characteristics of the invasive component; therefore, we cannot analyze the impacts of DCIS lesions on LN metastasis. In addition, potentially significant clinicopathological factors, such as vascular invasion, surgical margin status, adjuvant endocrine therapy, and targeted therapy, are not provided by the SEER database. Besides, HER2 status was not available before 2010 in the SEER database. The number of analyzed patients was very limited when HER2 status is considered, specifically in the propensity score matching analysis. Furthermore, due to the retrospective design of our study, there may be some inherent biases. Finally, agreement on chemotherapy between the SEER database and chart reviews proved to be moderate. ${ }^{29}$ The preferred approach would be to combine data from different sources to obtain more complete information.

\section{Conclusion}

Younger patients with high-grade disease tend to have LN metastasis in DCISM. DCISM patients are unlikely to benefit from adjuvant chemotherapy. Further studies and randomized trials should be performed before any appropriate suggestion can be made. 


\section{Acknowledgments}

This work is supported by National Natural Science Foundation of China (No. 81602471, No. 81672729), Zhejiang Provincial Natural Science Foundation of China under Grants(No. LY19H160055, LY19H160059, LY15H160020, Q16H160010), by grant from sub-project of China National Program on Key Basic Research Project (973 Program) (No. 2014CB744505).

\section{Author contributions}

Zhou J and Wang L designed the study; Zhou J and Chen $\mathrm{C}$ wrote the manuscript; Chen C, Huang S, Huang A, Jia Y, Wang $\mathrm{J}$, and Mao $\mathrm{M}$ analyzed the data and interpreted the results. All authors approved the final version to be published. All authors contributed to data analysis, drafting or revising the article, gave final approval of the version to be published, and agree to be accountable for all aspects of the work.

\section{Disclosure}

The authors report no conflicts of interest in this work.

\section{References}

1. Li Y, Zhang S, Wei X, Zhang J. The clinical features and management of women with ductal carcinoma in situ with microinvasion: A retrospective Cohort study. Int J Surg. 2015;19:91-94.

2. Bianchi S, Vezzosi V. Microinvasive carcinoma of the breast. Pathol Oncol Res. 2008;14(2):105-111.

3. Edge S, Byrd DR, Compton CC, Fritz AG, Greene F, Trotti A, editors. AJCC Cancer Staging Manual. 7th ed. New York: Springer; 2010.

4. Parikh RR, Haffty BG, Lannin D, Moran MS. Ductal carcinoma in situ with microinvasion: prognostic implications, long-term outcomes, and role of axillary evaluation. Int J Radiat Oncol Biol Phys. 2012;82(1):7-13.

5. Gray RJ, Mulheron B, Pockaj BA, Degnim A, Smith SL. The optimal management of the axillae of patients with microinvasive breast cancer in the sentinel lymph node era. Am J Surg. 2007;194(6):845-849.

6. van La Parra RF, Ernst MF, Barneveld PC, Broekman JM, Rutten MJ, Bosscha $\mathrm{K}$. The value of sentinel lymph node biopsy in ductal carcinoma in situ (DCIS) and DCIS with microinvasion of the breast. Eur J Surg Oncol. 2008;34(6):631-635.

7. Lee SK, Cho EY, Kim WW, et al. The prediction of lymph node metastasis in ductal carcinoma in situ with microinvasion by assessing lymphangiogenesis. J Surg Oncol. 2010;102(3):225-229.

8. Tunon-de-Lara C, Giard S, Buttarelli M, et al. Sentinel node procedure is warranted in ductal carcinoma in situ with high risk of occult invasive carcinoma and microinvasive carcinoma treated by mastectomy. Breast J. 2008;14(2):135-140.

9. Guth AA, Mercado C, Roses DF, Darvishian F, Singh B, Cangiarella JF. Microinvasive breast cancer and the role of sentinel node biopsy: an institutional experience and review of the literature. Breast J. 2008;14(4):335-339.

10. Wasserberg N, Morgenstern S, Schachter J, Fenig E, Lelcuk S, Gutman H. Risk factors for lymph node metastases in breast ductal carcinoma in situ with minimal invasive component. Arch Surg. 2002; 137(11):1249-1252.

11. Yi M, Krishnamurthy S, Kuerer HM, et al. Role of primary tumor characteristics in predicting positive sentinel lymph nodes in patients with ductal carcinoma in situ or microinvasive breast cancer. Am J Surg. 2008;196(1):81-87.
12. Matsen CB, Hirsch A, Eaton A, et al. Extent of microinvasion in ductal carcinoma in situ is not associated with sentinel lymph node metastases. Ann Surg Oncol. 2014;21(10):3330-3335.

13. Wang J, Mittendorf EA, Sahin AA, et al. Outcomes of sentinel lymph node dissection alone vs. axillary lymph node dissection in early stage invasive lobular carcinoma: a retrospective study of the surveillance, epidemiology and end results (SEER) database. PLoS One. 2014; 9(2):e89778

14. Wu Q, Ding X, Li J, et al. Surgical treatment in Paget's disease with invasive ductal carcinoma: an observational study based on SEER. Sci Rep. 2017;7:45510.

15. Sopik V, Sun P, Narod SA. Impact of microinvasion on breast cancer mortality in women with ductal carcinoma in situ. Breast Cancer Res Treat. 2018;167(3):787-795.

16. Wang W, Zhu W, du F, Luo Y, Xu B. The demographic features, clinicopathological characteristics and cancer-specific outcomes for patients with microinvasive breast cancer: A SEER database analysis. Sci Rep. 2017;7(1):42045.

17. Orzalesi L, Casella D, Criscenti V, et al. Microinvasive breast cancer: pathological parameters, cancer subtypes distribution, and correlation with axillary lymph nodes invasion. Results of a large single-institution series. Breast Cancer. 2016;23(4):640-648.

18. Kapoor NS, Shamonki J, Sim MS, Chung CT, Giuliano AE. Impact of multifocality and lymph node metastasis on the prognosis and management of microinvasive breast cancer. Ann Surg Oncol. 2013;20(8): 2576-2581.

19. Pimiento JM, Lee MC, Esposito NN, et al. Role of axillary staging in women diagnosed with ductal carcinoma in situ with microinvasion. J Oncol Pract. 2011;7(5):309-313.

20. Fang Y, Wu J, Wang W, et al. Biologic behavior and long-term outcomes of breast ductal carcinoma in situ with microinvasion. Oncotarget. 2016;7(39):64182-64190.

21. Lyons JM 3rd, Stempel M, Van Zee KJ, Cody HS 3rd. Axillary node staging for microinvasive breast cancer: is it justified? Ann Surg Oncol. 2012;19(11):3416-3421.

22. Meretoja TJ, Heikkilä PS, Salmenkivi K, Leidenius MH. Outcome of patients with ductal carcinoma in situ and sentinel node biopsy. Ann Surg Oncol. 2012;19(7):2345-2351.

23. Murphy CD, Jones JL, Javid SH, et al. Do sentinel node micrometastases predict recurrence risk in ductal carcinoma in situ and ductal carcinoma in situ with microinvasion? Am J Surg. 2008;196(4):566-568.

24. Gojon H, Fawunmi D, Valachis A. Sentinel lymph node biopsy in patients with microinvasive breast cancer: a systematic review and meta-analysis. Eur J Surg Oncol. 2014;40(1):5-11.

25. Niu HF, Wei LJ, Yu JP, et al. Is adjuvant chemotherapy necessary for patients with microinvasive breast cancer after surgery? Cancer Biol Med. 2016;13(1):142-149.

26. National Comprehensive Cancer Network. NCCN clinical practice guidelines in oncology: breast cancer, version 2; 2018.

27. Kapoor NS, Shamonki J, Sim MS, Chung CT, Giuliano AE. Impact of multifocality and lymph node metastasis on the prognosis and management of microinvasive breast cancer. Ann Surg Oncol. 2013; 20(8):2576-2581.

28. Piccart M, Rutgers E, Veer LV, et al. Primary analysis of the EORTC 10041/BIG 3-04 MINDACT study: a prospective, randomized study evaluating the clinical utility of the 70-gene signature (MammaPrint) combined with common clinical-pathological criteria for selection of patients for adjuvant chemotherapy in breast cancer with 0 to 3 positive nodes [abstract]. In: Proceedings of the 107th Annual Meeting of the American Association for Cancer Research; April 16-20, 2016; New Orleans, LA. Philadelphia (PA): AACR; Cancer Res. 2016;76(14 Suppl): Abstract nr CT039.

29. Du XL, Key CR, Dickie L, et al. Information on chemotherapy and hormone therapy from tumor registry had moderate agreement with chart reviews. J Clin Epidemiol. 2006;59(1):53-60. 


\section{Publish your work in this journal}

OncoTargets and Therapy is an international, peer-reviewed, open access journal focusing on the pathological basis of all cancers, potential targets for therapy and treatment protocols employed to improve the management of cancer patients. The journal also focuses on the impact of management programs and new therapeutic agents and protocols on

patient perspectives such as quality of life, adherence and satisfaction. The manuscript management system is completely online and includes a very quick and fair peer-review system, which is all easy to use. Visit http://www.dovepress.com/testimonials.php to read real quotes from published authors.

Submit your manuscript here: http://www.dovepress.com/oncotargets-and-therapy-journal 